\title{
Genes and regulatory networks involved in persistence of Mycobacterium tuberculosis
}

\author{
WANG XiaoZhen ${ }^{1}$, WANG HongHai ${ }^{2} \&$ XIE JianPing ${ }^{1,2 *}$ \\ ${ }^{1}$ Institute of Modern Biopharmaceuticals, State Key Laboratory Breeding Base of Eco-Environment and Bio-Resource of the Three Gorges \\ Area, School of Life Sciences, Southwest University, Chongqing 400715, China; \\ ${ }^{2}$ State Key Laboratory of Genetic Engineering, Institute of Genetics, School of Life Science, Fudan University, Shanghai 200433, China
}

Received July 15, 2010; accepted September 6, 2010; published online January 25, 2011

\begin{abstract}
The causative agent of tuberculosis, Mycobacterium tuberculosis, is one of the most successful of human pathogens. It can evade the host immune response and establish a persistent infection or enter a dormant state within the host which can be reactivated if the host becomes immuno-compromised. Both of these features are major obstacles to tuberculosis eradication. Dormancy and reactivation of $M$. tuberculosis are tightly coordinated dynamic processes involving numerous genes and their products. Molecular mechanisms underlying M. tuberculosis persistence may provide an opportunity for the discovery of effective drug targets for tuberculosis control. Here, we review the genes required for $M$. tuberculosis persistence and propose a regulatory network for the action of these genes using text mining. This should provide fresh insights into the persistence mechanisms of M. tuberculosis and suggest candidates for new drug targets and immune intervention.
\end{abstract}

Mycobacterium tuberculosis, persistent infection, dormancy, network

Citation: Wang X Z, Wang H H, Xie J P. Genes and regulatory networks involved in persistence of Mycobacterium tuberculosis. Sci China Life Sci, 2011, 54: 300-310, doi: 10.1007/s11427-011-4134-5

The causative agent of tuberculosis, Mycobacterium tuberculosis, is one of the most successful of human pathogens, but much of its biology remains unclear. Archaeological findings show that M. tuberculosis is at least as old as human beings on earth. The application of a series of serendipitously discovered antibiotics from the 1940s onwards halted the rampant spread of $M$. tuberculosis and the incidence rate of TB declined. However, during the late 1980s, drug-resistant $M$. tuberculosis strains began to emerge and are now found worldwide. The World Health Organization has issued two major alerts regarding the seriousness of tuberculosis. Approximately eight million individuals develop active tuberculosis annually, and two million die from the disease. HIV co-infection, widespread application of immunosuppressive agents and an aging population often

*Corresponding author (email: jianpingxiefudan@gmail.com) exacerbate the difficulties of tuberculosis control. The vast majority of individuals infected with $M$. tuberculosis exhibit no overt symptoms of active disease. Most are latent carriers with a $2 \%-23 \%$ lifetime risk of developing reactivation tuberculosis [1]. Once the host immune function wanes, asymptomatic persistent infection may become active tuberculosis. However, little is known about the molecular mechanisms underlying $M$. tuberculosis persistence and reactivation. No clinical drugs specifically targeting persistent $M$. tuberculosis are available. A lack of well-recognized animal models or in vitro models to study the persistence of M. tuberculosis further complicates this problem. However, features of $M$. tuberculosis may be found via models simulating one or two aspects of M. tuberculosis persistence and reactivation. It is generally recognized that compounds capable of shortening the duration or accelerating activation of persistent $M$. tuberculosis may point to anti-tuberculosis 
therapies. In this study we propose a regulatory network of M. tuberculosis genes underlying persistence using text mining, which we believe may elucidate persistence mechanisms and novel drug targets, and facilitate discovery and identification of new immune intervention strategies for tuberculosis control.

\section{M. tuberculosis genes implicated in persistent infection and their functions}

Persistence of $M$. tuberculosis necessitates the coordinated expression of numerous virulence determinants, including those involved in intermediary and secondary metabolism, cell wall processes, stress responses, and signal transduction pathways. However, only a handful of these genes have been experimentally demonstrated and not all of them are present in all currently available models. This suggests that the models should also be further refined.

\subsection{Metabolic pathway and persistence}

Swift adaptation to environmental cues is a typical feature of the most successful M. tuberculosis strains. Many studies have shown that $M$. tuberculosis metabolic pathways are rapidly altered to acclimatize to the macrophage milieu. Pinpointing such alterations will be useful in understanding the organism's regulatory processes and leading to the discovery of novel drug targets. M. tuberculosis genes impli- cated in persistence which have been reported in the literature are shown in Table 1.

\subsection{1 icl, gcvB and glyoxylate shunt}

Fatty acids are widely regarded as a major source of carbon and energy for persistent M. tuberculosis [2]. The anaplerotic pathway for the tri-carboxylic acid (TCA) cycle afforded by the glyoxylate shunt is vital for the survival of the bacteria [18]. The major source of carbon is furnished by $\beta$-oxidation of fatty acids when the generation of pyruvate from glycolysis is dramatically reduced. The M. tuberculosis icl gene, which encodes the isocitrate lyase, is required for persistence. Isocitrate lyase, the initial enzyme in the glyoxylate cycle, catalyzes the conversion of isocitrate to glyoxylate and succinate [1]. This enzyme plays a pivotal role in the regulation of isocitrate flux at the branch point between the glyoxylate shunt and the TCA cycle [19]. The M. tuberculosis $\Delta i c l$ mutant is markedly attenuated for survival in activated but not resting macrophages [2]. The disruption of $\mathrm{icl}$ attenuates persistence and virulence of $M$. tuberculosis in immune-competent mice without affecting bacterial growth during the acute stage of infection, and hence is also called a "persistence factor" [19]. However, this result has been challenged recently by studies that have found no strict correlation between the icl expression and whether the fatty acids are the sole carbon source (personal communication with Dr. Shi LanBo from New Jersey Medical School, USA). $g c v B$ encodes a putative glycine dehydrogenase that catalyzes the reductive amination of

Table 1 Genes implicated in Mycobacterium tuberculosis persistence

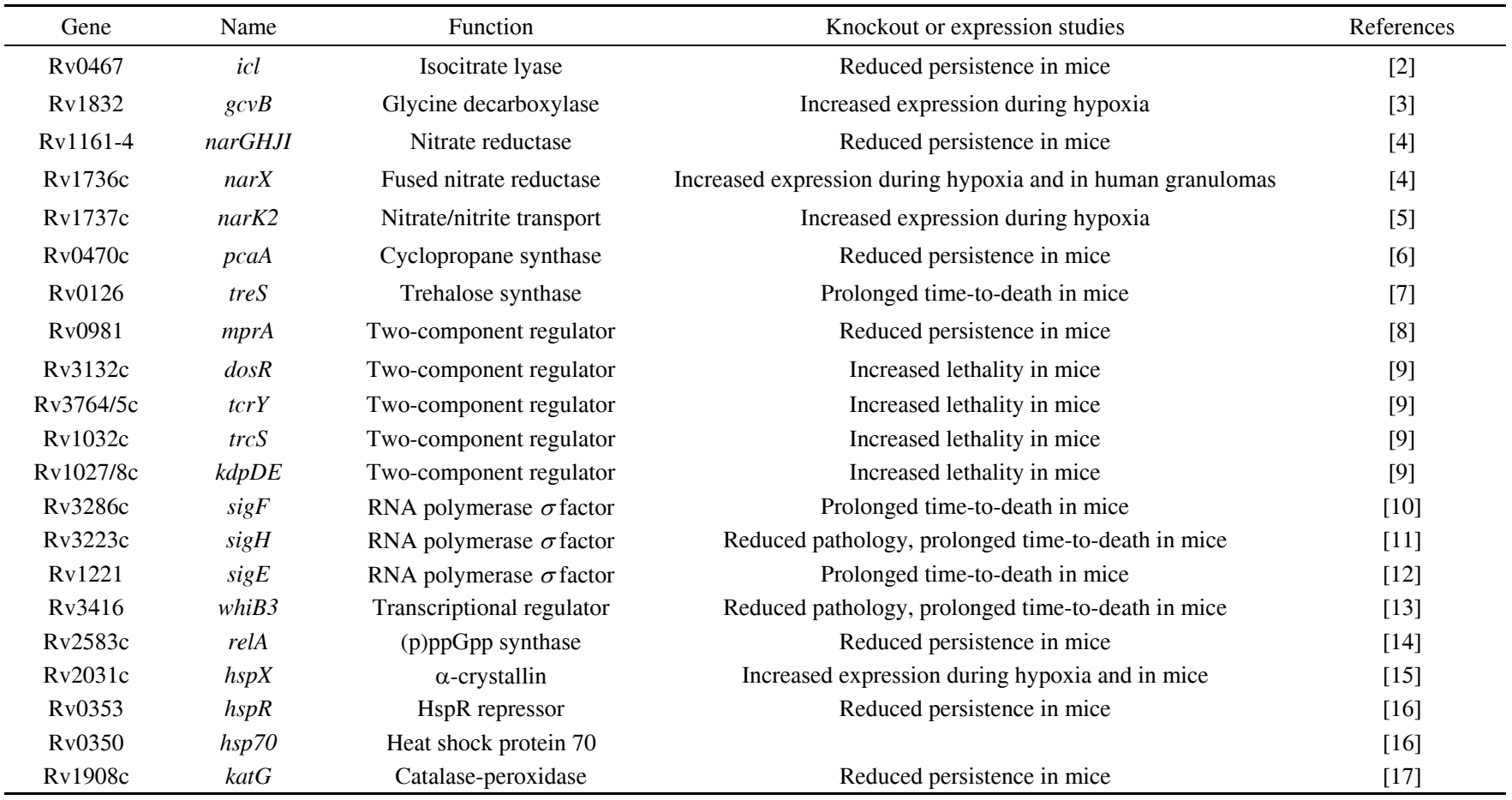


glyoxylate, concomitantly oxidizing NADH to NAD. Its activity is significantly up-regulated upon the entry of $M$. tuberculosis into persistence in vitro [3].

\subsection{2 narGHJI, narK2, narX and nitrate respiration}

Many bacteria can use nitrate instead of oxygen as a terminal electron acceptor to maintain the proton motive gradient to sustain growth under anaerobic conditions. M. tuberculosis is the most active nitrate-reducer among all slow growing mycobacteria [20]. The activity of nitrate reductase encoded by $M$. tuberculosis narGHJI is low during aerobic growth, but sharply up-regulated by hypoxia [21]. This induction might serve a respiratory function for M. tuberculosis adaptation to non-replicating persistence [20]. M. bovis BCG lacking anaerobic nitrate reductase $(\Delta$ narG $)$ fails to persist in the lungs, livers, and kidneys of immune-competent $\mathrm{BALB} / \mathrm{c}$ mice, but persistence and growth of BCG in the spleens of these mice appear largely unaffected, indicating that the role of the enzyme in pathogenesis is tissue-specific [4]. The marked activity increase is because of the induction of the nitrate transporter encoded by narK 2 instead of the up-regulation of the narGHJI [22]. Nitrite production increases markedly in M. tuberculosis cultures grown with nitrate during hypoxic adaptation. It may be secreted into the environment by NarK2, a nitrite-extrusion protein that has been shown to be up-regulated in response to hypoxia $[5,23]$. The $M$. tuberculosis gene narX encodes a "fused nitrate reductase", a protein with homology to parts of the NarG, NarJ, and NarI proteins. Its real function is unknown. However, the nitrate reductase activity of the $\Delta$ narX mutant was comparable to that of the wild type [24].

\subsection{Cell wall and persistence}

The cell wall of Mycobacterium spp. contains unique glycolipids, mycolic acids and long-chain branched fatty acids that are covalently or non-covalently attached to the cell surface [1]. Cell wall components are M. tuberculosis important factors regulating persistence in M. tuberculosis. Cell wall determinants implicated in $M$. tuberculosis pathogenesis are well documented, and include phthiocerol dimycocerosate, phenolic glycolipid, oxygenated mycolate, mycolic acid, and mycolic acid cyclopropanation [25].

\subsection{1 pcaA}

$p c a A$ encodes a cyclopropane synthase, an enzyme required for mycolic acid cyclopropane ring synthesis in the cell wall of both the tuberculosis vaccine, bacillus Calmette-Guérin (BCG) and M. tuberculosis [6]. Mycolic acids are longchain branched fatty acids that are covalently attached to the cell wall or present in the form of a toxic glycolipid trehalose dimycolate. Cyclopropane rings are a modification of membrane lipids that are found in diverse bacterial species. The $p c a A$ gene is required for long-term persistence and virulence of $M$. tuberculosis and the deletion of pcaA re- duces the ability of $M$. tuberculosis to undergo long-term persistence in the host. The $\triangle p c a A$ mutant can be eliminated faster from mice although it does also grow more rapidly than wild-type $M$. tuberculosis during acute stages of infection [6]. Mice infected with the $\triangle p c a A$ mutant exhibit a significant increase in time-to-death compared to mice infected with wild-type $M$. tuberculosis [1]. The contribution of pcaA expression to $M$. tuberculosis persistence remains unclear. The inability to attach cyclopropane rings to cell wall mycolic acids may alter the host antigen presentation. The $p c a A$ gene is required for the synthesis of the cord factor in the $M$. tuberculosis cell wall. Inhibition of cyclopropanation of cell wall mycolic acid can result in a change in lipid fluidity and cell wall permeability, and therefore a change in resistance to reactive oxygen intermediates (ROIs) and reactive nitrogen intermediates (RNIs) [23].

\subsection{2 treS}

Trehalose, encoded by treS, presents as a free disaccharide in the cytoplasm of mycobacteria and a component of cell-wall glycolipids implicated in tissue damage associated with mycobacterial infection [26]. It can participate in the biosynthesis of the cell wall of replicating M. tuberculosis and function as a stress protectant in non-replicating bacteria during exposure to a range of environmental stresses, including heat shock, dehydration, and hypoxia [7]. Trehalose 6,6'-dimycolate (TDM), also known as cord factor, occurs in the outer envelope of all mycobacteria and is the most abundant extractable toxic lipid on the surface of $M$. tuberculosis. TDM is capable of inducing granulomas that are similar to those in human tuberculosis, increasing chemokine and cytokine production and influencing the morphology of mycobacterial colonies [27-29]. On the surface of $M$. tuberculosis, TDM protects the bacteria from killing within macrophages by blockage of phagosomelysosome fusion and decreasing the acidification of phagosomes [30].

\subsection{Signal transduction and persistence}

Signal transduction is indispensable for $M$. tuberculosis persistence within granulomas for handling various stresses including hypoxia, nutrient limitation, reactive oxygen and nitrogen intermediates, low $\mathrm{pH}$, alveolar surfactants, and toxic proteins and fatty acids. $M$. tuberculosis two-component signaling systems and stress responsive sigma factors have been extensively studied.

\subsubsection{Two-component systems}

Two-component systems (TCS) usually consist of histidine kinase sensor proteins and cognate cytoplasmic response regulator proteins. Eleven complete and several orphaned two-component systems are present in the M. tuberculosis genome, many of which are implicated in persistent $M$. tuberculosis infection and are capable of regulating gene ex- 
pression to adapt $M$. tuberculosis to environmental conditions that simulate the intracellular milieu.

(i) $m p r A$

mprAB encodes a $M$. tuberculosis two-component system that is important for virulence during the persistent stage of infection [31]. M. tuberculosis mprAB is induced in response to nutrient starvation and low concentrations of sodium dodecyl sulfate (SDS). Inactivation of $m p r A$ enhances M. tuberculosis survival in resting macrophages but impairs M. tuberculosis persistence in lungs and spleens of mice [8]. The disruption of mprA is tissue-specific and affects the growth of $M$. tuberculosis during multiple stages of the infection process [8]. Compared to the parental strain, the $M$. tuberculosis $\triangle m p r A B$ mutant is more resistant to SDS and grows better in human peripheral blood monocytes [32]. MprA binds to conserved motifs in the upstream of both $\operatorname{sig} B$ and $\operatorname{sig} E$ in vitro and directly regulates the in vivo expression of $M$. tuberculosis $\operatorname{sig} B$ and $\operatorname{sig} E$ [31]. $\operatorname{mpr} A B$ is also regulated by sigma factors. For example, SDS exposure induces sigE expression, ensuing activation of 23 genes, including sigB, mprA, and $m p r B$ [32,33]. MprAB has either a directly positive or negative effect on the expression of acr2, which encodes a small molecule chaperone [34]. MprA auto-regulates its own expression and that of downstream gene pepD, which encodes trypsin-like serine protease [35]. During exponential growth and/or in the presence of SDS, the disruption of mprA also up-regulates multiple stress-associated genes of the DosR, SigD and IdeR regulons, including $r p f$ (resuscitation-promoting factor), narK2 (nitrate/nitrite transporter), pks4 (polyketide synthase), mmpL10 (fatty acid transport), and iron responsive genes such as hisE (phosphoribosyl-ATP pyrophosphatase), $m b t B$ and $m b t D$ (mycobactin synthetase), $f d x A$ (ferredoxin), and Rv2123 (PPE37) [32].

(ii) $\operatorname{dos} R$

The Dos two-component system consists of two sensor histidine kinases, DosS and DosT, and the cognate response regulator DosR. DosS functions as a redox sensor and DosT functions as a hypoxia sensor [36]. Hypoxia, NO, CO, S-nitrosoglutathione (GSNO), ethanol and $\mathrm{H}_{2} \mathrm{O}_{2}$ can all induce the expression of $M$. tuberculosis dosR gene [37]. Parish et al. [9] found that the growth of the $\Delta d o s R$ mutant was greater than that of the wild-type strain in IFN-r-activated macrophages, and SCID mice infected intravenously with the $\Delta d o s R$ mutant died more rapidly than mice infected with wild-type $M$. tuberculosis. This is the first report of an increase in mycobacterial virulence following targeted gene deletion. However, Malhotra et al. [38] found that a mutant expressing a truncated DosR protein showed reduced pathology and spleen bacillary burdens in subcutaneously infected guinea pigs. Recently, another study demonstrated that the disruption of $\operatorname{dos} R$ led to attenuation in resistant BALB/c and C57BL/6 mice and guinea pigs [39]. This is consistent with previously reported findings of attenuation in guinea pigs, but is contrary to findings of hypervirulence in mice. Genes induced by DosR include $h s p X$ (14 kD heat shock protein), $f d x A$ (ferredoxin), $p f k B$ (phosphofructokinase), narK2 (nitrate/nitrite transporter), $n r d Z$ (ribonucleoside diphosphate reductase), $c t p F$ (metal cation transporter P-type ATPase), $b f r B$ (bacterioferritin), $\operatorname{nar} X$ (fused nitrate reductase), and ots $B$ (trehalose-6-phosphate phosphatase) [40].

\section{(iii) $k d p D E, t c r Y$ and $\operatorname{trc} S$}

Kdp signal transduction, mediated primarily by $\mathrm{K}^{+}$concentration, is a primary bacterial response to environmental osmotic stress. E. coli regulates its cytoplasmic $\mathrm{K}^{+}$content through the $k d p F A B C$ and $k d p D E$ operons to tackle the osmotic stress. The $k d p F A B C$ operon encodes the $\mathrm{K}^{+}$-transporting P-type ATPase [41]. The $k d p$ genes encode for components of a mycobacterial signaling pathway by demonstrating the $\mathrm{K}^{+}$dependence of $k d p F A B C$ expression in both M. tuberculosis and Mycobacterium smegmatis [42]. A yeast two-hybrid screen shows that the sensing module of $M$. $t u$ berculosis KdpD interacts specifically with two membrane lipoproteins, LprJ and $\mathrm{LprF}$ [42]. The gene $t c r X$ is markedly induced under iron-limitation and $t c r X$ expression has been observed during the post-infection period in human macrophages infected by M. tuberculosis $[43,44]$. Consistent with the $\operatorname{dos} R$ gene, SCID mice infected intravenously with three of the M. tuberculosis mutants (deletions of $\operatorname{tcr} X Y, \operatorname{trcS}$, and $k d p D E$ ) die more rapidly than mice infected with wild-type strains [9].

\subsubsection{Sigma factors}

The sigma factor reversibly associates with the prokaryotic core RNA polymerase, forming the RNA poly-holoenzyme, and is responsible for promoter recognition. The M. tuberculosis genome harbors 13 different putative sigma factors. Several extracytoplasmic function sigma factors are induced upon the exposure of $M$. tuberculosis to various environmental stresses.

(i) $\operatorname{sig} F$

The $M$. tuberculosis sigF gene is strongly induced upon exposure to nitrogen limitation, cold shock, nutrient starvation and entry into stationary phase $[45,46]$. The growth rate of the M. tuberculosis $\Delta$ sigF mutant is identical to that of the wild-type strain in culture and human macrophages during exponential phase, but the mutants grow to a higher density than the wild-type strain in stationary phase [10]. In $\mathrm{BALB} / \mathrm{c}$ mice, the $\Delta s i g F$ mutant and wild-type strains have nearly identical growth rates and similar degrees of granulomatous inflammation during the early stage after infection, but after eight weeks, the $\Delta s i g F$ mutant persists in the lung and shows fewer lesions and less inflammation, while the wild type continues to proliferate slowly [47]. DNA microarray data shows that genes down-regulated significantly in the $M$. tuberculosis $\Delta$ sig $F$ mutant strain during exponen- 
tial phase are mostly involved in fatty acids and phospholipid metabolism, detoxification and protein folding, such as acpP (acyl carrier protein), fabD (malonyl CoA-acyl carrier protein), pks16 (polyketide synthase pKS16), efpA (efflux protein), groES (10 kD chaperonin), hsp (Hsp20 homologue), glnAl (glutamine synthetase), trxC (thioredoxin), kasA (3-oxoacyl-(acyl carrier protein) synthase), $m d h$ (malate dehydrogenase), echA6 (enoyl-CoA hydratase/isomerase), Rv0297 (PE-PGRS5), and Rv0285 (PE5) [47]. Notably, the $a c p C$ gene, which encodes alkyl hydroperoxidase and is involved in isoniazid resistance, is strongly down-regulated by the loss of $\operatorname{sig} F$. Genes down-regulated by the deletion of $\operatorname{sig} F$ during the stationary phase include those involved in energy metabolism, nucleotide synthesis, central intermediary metabolism and cell envelope synthesis, such as esx $Q$ (esat-6-like protein esxQ), $\operatorname{trp} G$ (glutamine amidotransferase), mmpLl (transmembrane transport protein), $n d h A$ (NADH dehydrogenase), $n r d Z$ (ribonucleoside-diphosphate reductase), bfrA (bacterioferritin), $p k n B$ (serine/threonine protein kinase), ufaAl (cyclopropane-fatty-acyl-phospholipid synthase), pimB (mannose glycosyltransferase), fadD30 (acyl-CoA synthetase), lipW (lipase), and fgd2 (glucose-6phosphate dehydrogenase) [47]. In addition, genes encoding several key transcriptional regulators such as TetR, GntR and MarR family regulators are also down-regulated. Moreover, the expression of $\operatorname{sig} B$ and $\operatorname{sig} C$ also appears to be SigF-dependent $[47,48]$. Overexpression of sigF results in significant up-regulation of genes encoding members of the MmpL family ( $m m p L 2, m m p L 5$ and $m m p L 11$ ), lipoproteins (lprA, lprE, lprK) and other genes such as pks14, murA, $\operatorname{sig} G, d n a A, e m b R$, ace $A b$, and trxA. The most highly up-regulated gene following $\operatorname{sig} F$ induction is $p h o Y 1$, which encodes a transcriptional regulatory protein homologous to PhoU proteins involved in the regulation of phosphate uptake [49].

(ii) $\mathrm{sigH}$

The expression of $M$. tuberculosis sigH is induced in response to heat shock and oxidative stress, and following uptake by macrophages [50]. The M. tuberculosis $\Delta$ sigH mutant is more sensitive than the wild-type strain to heat shock and to various oxidative stresses, but does not show decreased ability to grow inside macrophages [51]. In resistant C57BL/6 mice, the $\Delta$ sigH mutant achieves a high bacterial burden identical to those of wild-type strain in lung and spleen, but produces a blunted, delayed pulmonary inflammatory response, and recruits less CD4+ and CD8+ T cells to the lung [11]. In susceptible $\mathrm{C} 3 \mathrm{H}$ mice, the $\Delta$ sigH mutant again shows diminished immunopathology in contrast to wild-type bacilli [11]. Microarray expression profiles of the wild-type $M$. tuberculosis and the $\Delta$ sig $H$ mutant strain after exposure to the thiol-specific oxidizing agent diamide show that the deletion of $M$. tuberculosis sigH down-regulates cys $M$ (cysteine synthase B), cysA (sulphate transport ATP-binding protein), cys $W$ and cysT (sulphate transport system permease protein), papA4 (PKS-associated protein), chA12 (enoyl-CoA hydratase/isomerase protein), $h s p$ (heat-shock protein), $\operatorname{tr} x B 2, \operatorname{tr} x B$ (thioredoxin reductase), trxC (thioredoxin), murl (glutamate racemase), moeZ (molybdopterin biosynthesis protein), $r m l B$ (dTDP-glucose 4,6-dehydratase), rmlC (dTDP-4-dehydrorhamnose 3,5-epimerase), $\operatorname{clpB}$ (endopeptidase ATP binding protein), rpi (dTDP-4-dehydrorhamnose 3,5-epimerase), Rv0355c (PPE8), and $\operatorname{sig} B$ and $\operatorname{sig} E$ [51]. The inducible expression of $\operatorname{sig} B$ is dependent on SigE and SigH [50]. The expression of the heat shock genes $d n a K$ and $\operatorname{clpB}$ of the $\Delta s i g H$ mutant is down-regulated under heat stress [50].

(iii) $\operatorname{sig} E$

M. tuberculosis sigE shows increased expression following exposure to heat shock, SDS treatment, and oxidative stress, as well as after phagocytosis in macrophages [12]. The $M$. tuberculosis $\Delta$ sigE mutant is defective in its ability to grow inside macrophages but has identical bacterial burden to the wild-type strain in the lung of the $\mathrm{C} 3 \mathrm{H} / \mathrm{HeJ}$ mice [33]. Time-to-death analysis shows that $50 \%$ of $\mathrm{C} 3 \mathrm{H} / \mathrm{HeJ}$ mice and all the BALB/c-SCID mice infected with the $\triangle \operatorname{sig} E$ mutant strains die more slowly than the wild-type $M$. $t u$ berculosis [12]. DNA microarray data shows that 38 genes are down-regulated in $M$. tuberculosis $\Delta$ sigE mutant, among which five genes are involved in protein translation (tuf, rpsJ, rplD, rplP and infC), four in mycolic acid biosynthesis (fbpC2 (mycolyl transferase 85C), fbpB (mycolyl transferase 85B), fabD (acyl-carrier-protein S-malonyltransferase) and acpM (acyl carrier protein)), three are transcriptional regulators (Rv0287, $\operatorname{sig} B$ and $\operatorname{csp} A$ (cold shock protein $\mathrm{A})$ ), three are involved in electron transport (ctaE (cytochrome $\mathrm{C}$ oxidase subunit III), qcrA, qcrB (ubiQ-cytB reductase component)), two in acyl-CoA biosynthesis (aceE (pyruvate dehydrogenase E1) and fadD29 (acyl-CoA synthase)), and the last two are $g \ln A 1$ (glutamine synthase), sodA (superoxide dismutase) [33]. Only aceA (icl), Rv1130 and gltAl (citrate synthase 3 ) show a higher level of expression in the $\Delta \operatorname{sig} E$ mutant than in the wild-type strain [33].

\subsection{3 whiB}

M. tuberculosis whiB3 encodes a homolog of the Streptomyces coelicolor whiB gene, whose function is required for sporulation [52]. M. tuberculosis WhiB3 responds to signaling molecules $\mathrm{NO}$ and $\mathrm{O}_{2}$ via its $[4 \mathrm{Fe}-4 \mathrm{~S}]$ cluster and is also essential for surviving the nutrient starvation [53]. As a physiological regulator of virulence lipid anabolism, WhiB3 differentially modulates the assimilation of propionate into complex virulence polyketides under defined oxidizing and reducing conditions [54]. WhiB3 interacts with the principal sigma factor RpoV to affect host survival but is dispensable for in vivo growth of $M$. tuberculosis [13]. In addition, WhiB3 binds to $p k s 2$ and $p k s 3$ promoter DNA [54]. M. tuberculosis whiB3 is induced maximally during the early phase of mouse lung infection and whiB3 is induced in 
resting macrophages but repressed in IFN-r-activated macrophages [55]. Mice infected with the M. tuberculosis $\Delta$ whiB3 mutant have significantly longer mean survival time compared with mice challenged with wild-type strain. Remarkably, the bacterial organ burdens of both mutant and wild-type strains are identical during the acute and persistent phases of infection [13].

\subsection{Other genes required for persistent $M$. tuberculosis infection}

\subsection{1 $\mathrm{rel}_{\mathrm{Mtb}}$}

The stringent response utilizes hyperphosphorylated guanine [(p)ppGpp] as a signaling molecule to control bacterial gene expression involved in long-term survival under starvation conditions [14]. In gram-negative bacteria, RelA and SpoT are responsible for the synthesis of (p)ppGpp. M. $t u$ berculosis rel $_{M t b}$ encodes a single RelA or SpoT homolog, designated $\mathrm{Rel}_{\mathrm{Mtb}}$, a dual-function enzyme, for both (p)ppGpp synthesis and hydrolysis [56]. The M. tuberculosis $\Delta r l_{M t b}$ mutant is unable to synthesize (p)ppGpp in vitro on starvation and severely defective in the maintenance of long-term viability. The mutant strain displays a significantly slower growth rate than the wild type in synthetic liquid media but shows an identical growth rate to that of the wild type within a human macrophage-like cell line [14]. The ability of the $\Delta r e l_{M t b}$ mutant to sustain persistent infection in C57BL/6 mice infected via aerosol inoculation is severely impaired. Significant histopathologic differences are observed in lungs and spleens of mice infected with the $\Delta r e l_{M t b}$ mutant compared with controls [57]. A DNA microarray was carried out to analyze differentially expressed genes between the $M$. tuberculosis $\Delta$ rel $_{M t b}$ mutant and the wild-type strain. Genes displaying a higher level of expression in the wild type include $p b p A$ and $p b p B$ (penicillin-binding protein), mcel, mce $3 B$, mce 4 (cell invasion factor), narH (nitrate reductase $\beta$ chain), narI (nitrate reductase g chain), murC and murG (peptidoglycan synthesis protein), $f b p C$ (mycolyl transferase $85 \mathrm{C}), h s p X(14 \mathrm{kD}$ heat shock protein), lpqH (19 kD lipoprotein antigen precursor), pst $S 1$ (periplasmic phosphate-binding lipoprotein), esxA (6 kD early secretory antigenic target ESAT-6), Rv3144c (PPE52), Rv1646 (PE17), acpM, kasA, kasB, sigD, pks4, and rpf [57]. Genes whose expression levels are higher in the $\Delta r e l_{M t b} \mathrm{mu}-$ tant strain include groES (10 kD chaperone), groEL (heat shock protein 65), mmaA2 (methoxymycolic acid synthase 2), $f b p A, f b p B, f b p D$ (mycolyl transferase), lppO, lppS, lppU (lipoprotein), whiB3, eis, ompA, pks15 and genes encoding PE12, PE26, PE35, PPE27, PPE41, PE-PGRS42 [57].

\subsection{2 hspX}

The most dramatic transcriptional activation ( 80 -fold) by M. tuberculosis DosR during hypoxia is the induction of the hspX gene (acr), which encodes a 16-kD $\alpha$-crystallin-like heat shock protein. The HspX protein acts as a molecular chaperone, preventing thermally induced aggregation of other proteins, and may play a role in stabilizing cell structures during long-term survival of $M$. tuberculosis [58]. The M. tuberculosis $\Delta h s p X$ mutant exhibits increased growth in both resting and activated macrophages in vitro and following infection of BALB/c mice in vivo [15]. The increased growth of the $\Delta h s p X$ mutant in macrophages is equivalent to that of a $\triangle d o s R$ mutant (as measured by the number of CFU (colony forming unit)), which suggests that the increased number of CFU of the $\Delta d o s R$ mutant is largely due to loss of $h s p X$ expression [15]. Overexpression of $h s p X$ results in reduction in growth rates of both $M$. tuberculosis and $M y$ cobacterium smegmatis, and constitutive expression of $h s p X$ leads to a decreased growth rate for $M$. tuberculosis, suggesting that the $h s p X$ gene is associated with slow growth $[15,59]$.

\subsection{3 hsp70 and hspR}

Exposure of $M$. tuberculosis to increased temperature results in elevated expression of heat-shock genes. The HspR repressor inhibits expression of only a small number of $M$. tuberculosis genes, including the $h s p 70$ operon and the gene encoding the ATPase ClpB. Although the M. tuberculosis $\Delta h s p R$ mutant is fully virulent in the initial phase of infection, it is significantly impaired in its ability to persist during the persistent stage [16]. The deletion of $h s p R$ leads to constitutive overexpression of the Hsp70 proteins. The high level of the Hsp70 proteins within the cell might block some developmental program involved in mycobacterial adaptation and enhance the immune recognition of the $\Delta h s p R$ mutant.

\subsection{4 katG}

Many isoniazid-resistant strains of $M$. tuberculosis have deletions or missense mutations in the katG gene, which encodes the catalase-peroxidase. Transformation of functional $M$. tuberculosis katG genes into the avirulent $\mathrm{H} 37 \mathrm{RvINH}^{\mathrm{R}}$ strain restores the ability of this recombinant to multiply and persist in spleens of mice and guinea pigs [17].

\section{Construction of the gene regulatory network of persistent $M$. tuberculosis infection}

Most events within cells cannot be accomplished with a single gene or its product. Gene interactions underpin complex gene expression regulatory networks. Functional genomics methodologies including DNA microarray and mass spectrometry provide unprecedented opportunity to address the intricacy of gene regulatory networks. Gene regulatory networks depict complicated gene interactions and consist of many nodes (genes) and links among nodes (positive regulation or negative regulation). A gene regulatory net- 
work based on the genes and regulators previously reported to be implicated in M. tuberculosis persistence is shown in Figure 1.

This network indicates that genes encoding for sigma factors and two component systems play essential roles during the $M$. tuberculosis persistent infection stage. $M$. tuberculosis two component systems initially respond to various environmental stresses within the granuloma. Histidine kinase sensor proteins transmit stress signals to cytoplasmic response regulators to control the expression of sigma factors, and then mediate other genes to respond to environmental changes. The signal transduction pathway "mprB/A-sigE-relA" is a perfect example of this [60]. In principle, the signal transduction cascade can be intercepted at any one of these steps. Genes whose expressions are regulated include those encoding the $\mathrm{MmpL}$ protein family members, which are related to transport of lipids, such as mmpL1, mmpL2, mmpL5, mmpL10, mmpL11. MmpL-mediated lipid secretion both contributes to the intracellular survival of $M$. tuberculosis and also to the host-pathogen interaction that determines the ultimate outcome of infection [61]. Furthermore, there are many genes encoding PE/PPE/PEPGRS protein family members and genes from this family account for nearly $5 \%$ of coding capacity of M. tuberculosis. This protein family is required for virulence and persistence of $M$. tuberculosis and several members of this family localize to the cell surface and influence interactions between $M$. tuberculosis and the macrophages. PE-PGRS51 is a dominant antigen which is recognized by sera of latent carriers. In addition, this network also involves several salty ion transport genes, such as nitrate/nitrite transport gene narK2, sulfate transport genes cys $A$, cys $W$ and $\operatorname{cys} T$, and phosphate transport gene pstS1. Genes encoding for the Ag85 complex, which consists of three distinct trehalose dimycolyl transferases Ag85A, Ag85B and $\mathrm{Ag} 85 \mathrm{C}$, frequently appear in this network, including $f b p A, f b p B$, and $f b p C$. These antigens participate in mycolic acid biosynthesis and cell wall assembly. They also function as fibronectin-binding proteins (fbps) to attach to the macrophages. fbp genes are induced upon isoniazid treatment and suggestive of novel anti-tuberculosis drug targets [62].

Drug resistance of tuberculosis has recently emerged as a major public health challenge in China, and there has been an alarming increase in patients with multi-drug resistant tuberculosis (MDR-TB) and extensively drug resistant tuberculosis (XDR-TB). Resistance rates to isoniazid, rifampicin, and streptomycin are high. This network involves a large number of isoniazid resistance genes. The katG gene encodes catalase-peroxidase, an enzyme that converts isoniazid to an active form to inhibit the biosynthesis of the mycolic acid and cell wall. Mutations, deletions and insertions in the kat $G$ gene are responsible for isoniazid resistance. The kasA gene encodes 3-oxoacy-(acyl carrier protein)

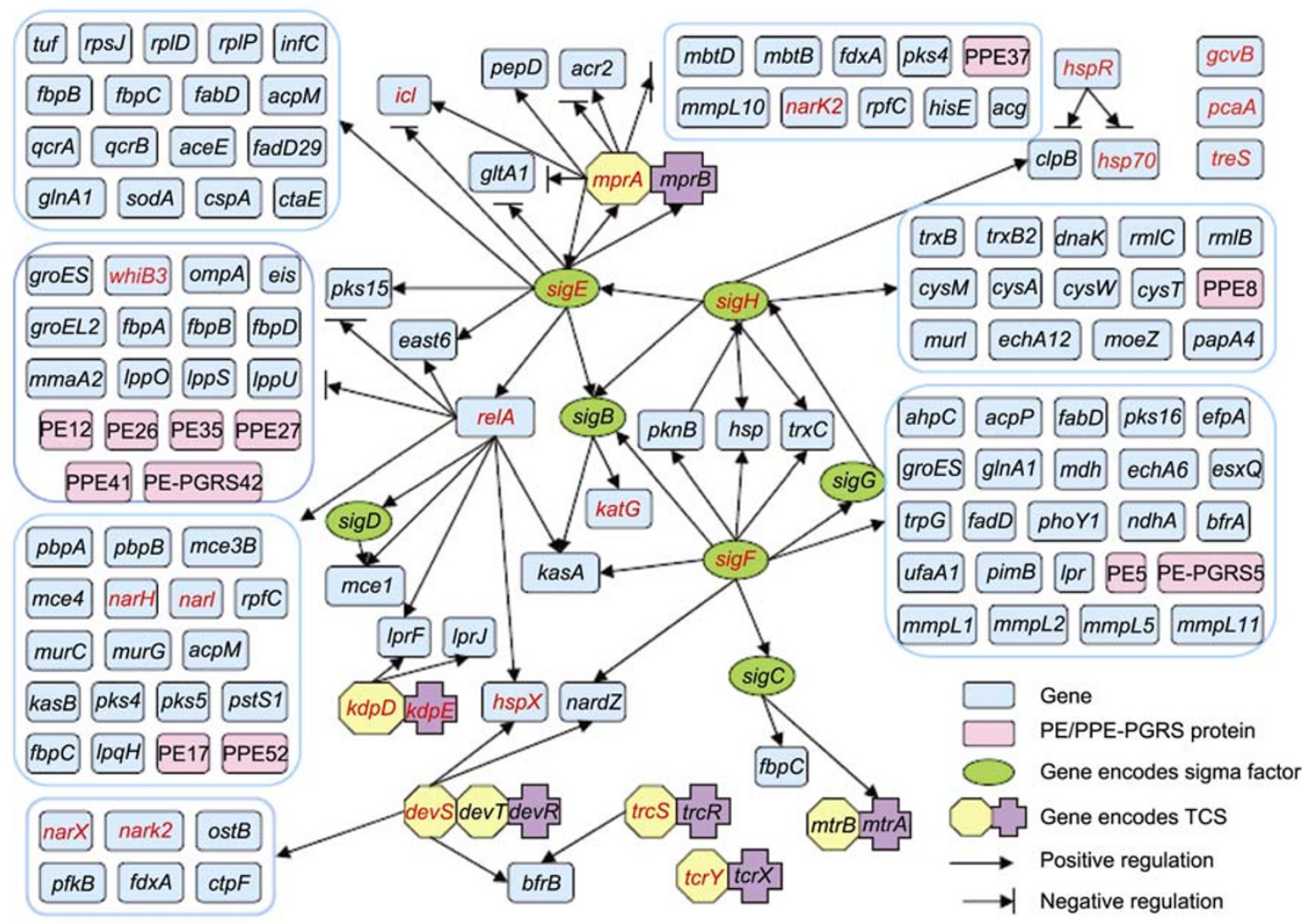

Figure 1 Gene regulatory network based on the genes and regulators reported to be implicated in $M$. tuberculosis persistence. The genes highlighted by red represent $M$. tuberculosis genes implicated in persistent infection which have been reported; the pink proteins represent genes which encode these proteins. 
synthase, a key enzyme involved in the biosynthesis of mycolic acid, and the mutation in the kasA gene is related to isoniazid resistance. The $a h p C$ gene encodes alkyl hydroperoxide reductase subunit $\mathrm{C}$ and the mutation of its promoter results in AhpC over-expression, thereby compensating the lack of catalase-peroxidase caused by $\operatorname{kat} G$ mutation. The $n d h$ gene encodes NADH dehydrogenase and mutations in the $n d h$ gene lead to defective activity of NADH dehydrogenase, increased NADH/NAD ${ }^{+}$ratio and inhibited peroxidation of isoniazid, suggesting that $n d h$ is associated with isoniazid resistance.

There are interactions among sigma factors and certain sigma factors can regulate other sigma factors, achieving an internal regulation of sigma factors. From this network we know that $\operatorname{sig} E, \operatorname{sig} H, \operatorname{sig} F$ are able to positively regulate $\operatorname{sig} B$ and $\operatorname{sig} B$ may auto-amplify its expression under certain conditions, suggesting that the complex interplay of sigma factors converges at $\operatorname{sig} B$ [63]. The expression of other sigma factors is unchanged after $\operatorname{sig} B$ induction, suggesting that $\mathrm{SigB}$ functions as an end regulator in the sigma factor regulatory network [63]. The deletion of $\operatorname{sig} B$ in $M$. tuberculosis results in its higher sensitivity to SDS, heat shock, oxidative stress and hypoxic conditions and over-expression of $\operatorname{sig} B$ significantly upregulates $k a t G, k a s A$, whiB2, and ideR $[63,64]$. Currently, the gene regulatory network of $M$. tuberculosis persistence is still far from complete. Also with such a complex organism, a small disturbance might well have a significant effect in this network and on the fate of the pathogen. Discovery of the pivotal nodes in this network will undoubtedly be important for the development of future intervention measures.

\section{Vaccine design and drug targets screening}

Long term persistence of $M$. tuberculosis is also a complex process requiring the coordinated expression of numerous M. tuberculosis virulence genes and adaptive immune evasion strategies. Understanding which genes and networks are implicated in M. tuberculosis persistence is instrumental in determining the underlying molecular mechanisms, and producing better drug targets for latent tuberculosis treatment.

\subsection{Inspiration for vaccine design}

The current tuberculosis vaccine BCG is largely ineffective in protecting adults against pulmonary tuberculosis. Most new generation tuberculosis vaccines are designed as prophylactic vaccines and are based on antigens that are expressed during the early phases of $M$. tuberculosis infection [65]. Persistent M. tuberculosis is unlikely to be targeted by such prophylactic vaccines, as they may not be effective in the already infected host during the late stage infection. One strategy is to develop therapeutic vaccines that can adequately activate
T-cell responses to late stage infection associated antigens. Such vaccines may target and help eliminate dormant or slowly replicating $M$. tuberculosis, thereby preventing later reactivation from latency [65]. Therapeutic vaccines alone or in combination with drugs may provide a more rapid and more effective strategy against persistent M. tuberculosis. Identifying genes required for persistent infection by $M$. tuberculosis and constructing gene regulatory networks should lead to the discovery of novel TB drug targets. The general nodes such as common genes and the hubs encoding two-component systems and sigma factors might be promising targets for vaccine design.

The heat shock protein HspX is required for persistence of M. tuberculosis within the macrophage. In the sera of tuberculosis patients, both T-cell and B-cell responses against HspX were found to be associated with persistent $M$. tuberculosis infection, indicating the importance of HspX as an antigenic target of immune responses during persistent infection. Subunit vaccines incorporating HspX and/or other latency antigens might be good vaccine candidates for latent tuberculosis [66]. Lowrie [67] demonstrated that post-therapy relapse of tuberculosis could be prevented by vaccinating the Cornell mice with a DNA vaccine encoding the mycobacterial Hsp65 heat shock protein, suggesting that the immune boost provided by vaccination promoted the complete eradication of $M$. tuberculosis from the tissues. Recombinant BCG expressing DosR can be aerobically cultured in vitro but fails to enter a non-replicating state, allowing evaluation of this strain as a latency-specific vaccine candidate [68]. In addition, the high survival rate in tissues with reduced lethality of the $M$. tuberculosis $\Delta$ sig $F$ mutant suggests that this class of mutant may be valuable as live-attenuated vaccines for tuberculosis. The $\Delta s i g F$ mutant strain appears to cause stronger immunity than BCG in the rabbit model, suggesting that alterations in the SigF regulon result in a superior set of immunogens with potentially more long-lived protection than those induced by BCG with potentially more long-lived protection [47].

\subsection{Inspiration for drug targets discovery}

Although some front-line antibiotics (isoniazid, ethambutol, pyrazinamide, and rifampicin) are still reasonably effective at killing $M$. tuberculosis which is actively replicating in culture, these antibiotics are often ineffective in eliminating M. tuberculosis during persistent stages of infection [1]. The dearth of specific drugs to target persistent M. tuberculosis is the major barrier to developing effective short-term treatment. Research into tuberculosis has placed an inappropriate emphasis on the need to discover genetic "essentiality" for any target, based on the misplaced assumption that genetic knockouts can be phenotypically copied by small-molecule inhibitors of metabolic pathways. We need to shift the emphasis from the discovery of essential genes to identifying the subtle holistic interactions between path- 
ways and gene products that are required for survival in the dynamic host milieu. These interactions constitute a gene regulatory network; central nodes in this network are likely to be metabolic breakpoints that could be considered as drug targets. For example, DosR is a key transcriptional regulator for coordinating the response to host immune attack which eventually results in the entry into the non-replicating state. Therefore, it is widely recognized as a potential drug target against latent tuberculosis. However, by inhibiting $\operatorname{dos} R$, hypervirulent strains of $M$. tuberculosis were produced. This precludes precise prediction of the effect of $d o s R$ inactivation on shortening the duration of tuberculosis treatment [69].

\section{Concluding remarks}

Significant advances have been made in identifying $M$. $t u$ berculosis "persistence factors", but it is not yet clear whether targeting these persistence factors with new drugs will fulfill the expectation of killing M. tuberculosis in vivo, nor is it clear whether persistence factors that are essential in the mouse model will likewise be important in human disease. A major challenge for future research will be the formulation of models of persistent infection relevant to human infection and replicating the behavior of $M$. tuberculosis in its natural niche [70]. Reporter enzyme fluorescence (REF) allows real-time imaging of pulmonary infections and rapid quantification of $M$. tuberculosis in living animals. This technology is based on near-infrared (NIR) fluorogenic substrates for $\beta$-lactamase, an enzyme expressed by $M$. tuberculosis, but not by their eukaryotic hosts [71]. Gene regulatory network study is an emerging area for which various models and computational tools are now available. Construction of gene regulatory networks will help us to understand the interactions among genes required for $M$. tuberculosis persistent infection, to study gene functions from a global perspective and to discover key therapeutic targets against tuberculosis. Much endeavor in this field should lead to better control of TB.

This work was supported by the National Key Infectious Disease Project (Grant Nos. 2008ZX10003-006 and 2008ZX10003-001), the Excellent PhD Thesis Fellowship of Southwest University (Grant Nos. kb2009010 and ky2009009), the Fundamental Research Funds for the Central Universities (Grant No. XDJK2009A003), the Natural Science Foundation Project of CQ CSTC (Grant No. CSTC, 2010BB5002), and the National Natural Science Foundation of China (Grant No. 81071316).

1 Zahrt T C. Molecular mechanisms regulating persistent Mycobacterium tuberculosis infection. Microbes Infect, 2003, 5: 159-167

2 McKinney J D, Honer zu Bentrup K, Munoz-Elias E J, et al. Persistence of Mycobacterium tuberculosis in macrophages and mice requires the glyoxylate shunt enzyme isocitrate lyase. Nature, 2000, 406: 735-738

3 Wayne L G, Lin K Y. Glyoxylate metabolism and adaptation of Mycobacterium tuberculosis to survival under anaerobic conditions. In- fect Immun, 1982, 37: 1042-1049

4 Fritz C, Maass S, Kreft A, et al. Dependence of Mycobacterium bovis $\mathrm{BCG}$ on anaerobic nitrate reductase for persistence is tissue specific. Infect Immun, 2002, 70: 286-291

5 Hutter B, Dick T. Analysis of the dormancy-inducible narK2 promoter in Mycobacterium bovis BCG. FEMS Microbiol Lett, 2000, 188: $141-146$

6 Glickman M S, Cox J S, Jacobs W R Jr. A novel mycolic acid cyclopropane synthetase is required for cording, persistence, and virulence of Mycobacterium tuberculosis. Mol Cell, 2000, 5: 717-727

7 Murphy H N, Stewart G R, Mischenko V V, et al. The OtsAB pathway is essential for trehalose biosynthesis in Mycobacterium tuberculosis. J Biol Chem, 2005, 280: 14524-14529

8 Zahrt T C, Deretic V. Mycobacterium tuberculosis signal transduction system required for persistent infections. Proc Natl Acad Sci USA, 2001, 98: 12706-12711

9 Parish T, Smith D A, Kendall S, et al. Deletion of two-component regulatory systems increases the virulence of Mycobacterium tuberculosis. Infect Immun, 2003, 71: 1134-1140

10 Chen P, Ruiz R E, Li Q, et al. Construction and characterization of a Mycobacterium tuberculosis mutant lacking the alternate sigma factor gene, sigF. Infect Immun, 2000, 68: 5575-5580

11 Kaushal D, Schroeder B G, Tyagi S, et al. Reduced immunopathology and mortality despite tissue persistence in a Mycobacterium tuberculosis mutant lacking alternative sigma factor, SigH. Proc Natl Acad Sci USA, 2002, 99: 8330-8335

12 Ando M, Yoshimatsu T, Ko C, et al. Deletion of Mycobacterium tuberculosis sigma factor E results in delayed time to death with bacterial persistence in the lungs of aerosol-infected mice. Infect Immun, 2003, 71: 7170-7172

13 Steyn A J, Collins D M, Hondalus M K, et al. Mycobacterium tuberculosis WhiB3 interacts with RpoV to affect host survival but is dispensable for in vivo growth. Proc Natl Acad Sci USA, 2002, 99: 3147-3152

14 Primm T P, Andersen S J, Mizrahi V, et al. The stringent response of Mycobacterium tuberculosis is required for long-term survival. J Bacteriol, 2000, 182: 4889-4898

$15 \mathrm{Hu}$ Y, Movahedzadeh F, Stoker N G, et al. Deletion of the Mycobacterium tuberculosis alpha-crystallin-like $h s p X$ gene causes increased bacterial growth in vivo. Infect Immun, 2006, 74: 861-868

16 Stewart G R, Snewin V A, Walzl G, et al. Overexpression of heat-shock proteins reduces survival of Mycobacterium tuberculosis in the chronic phase of infection. Nat Med, 2001, 7: 732-737

17 Li Z, Kelley C, Collins F, et al. Expression of katG in Mycobacterium tuberculosis is associated with its growth and persistence in mice and guinea pigs. J Infect Dis, 1998, 177: 1030-1035

18 Gould T A, van de Langemheen H, Munoz-Elias E J, et al. Dual role of isocitrate lyase 1 in the glyoxylate and methylcitrate cycles in $M y$ cobacterium tuberculosis. Mol Microbiol, 2006, 61: 940-947

19 Kumar R, Bhakuni V. Mycobacterium tuberculosis isocitrate lyase (MtbIcl): Role of divalent cations in modulation of functional and structural properties. Proteins, 2008, 72: 892-900

20 Wayne L G, Hayes L G. Nitrate reduction as a marker for hypoxic shiftdown of Mycobacterium tuberculosis. Tuber Lung Dis, 1998, 79: 127-132

21 Sohaskey C D, Modesti L. Differences in nitrate reduction between Mycobacterium tuberculosis and Mycobacterium bovis are due to differential expression of both narGHJI and narK2. FEMS Microbiol Lett, 2009, 290: 129-134

22 Sohaskey C D. Regulation of nitrate reductase activity in Mycobacterium tuberculosis by oxygen and nitric oxide. Microbiology, 2005, 151: 3803-3810

23 Honer zu Bentrup K, Russell D G. Mycobacterial persistence: Adaptation to a changing environment. Trends Microbiol, 2001, 9: 597605

24 Sohaskey C D, Wayne L G. Role of narK2X and narGHJI in hypoxic upregulation of nitrate reduction by Mycobacterium tuberculosis. J Bacteriol, 2003, 185: 7247-7256

25 Rao V, Gao F, Chen B, et al. Trans-cyclopropanation of mycolic ac- 
ids on trehalose dimycolate suppresses Mycobacterium tuberculosis-induced inflammation and virulence. J Clin Invest, 2006, 116: 1660-1667

26 De Smet K A, Weston A, Brown I N, et al. Three pathways for trehalose biosynthesis in mycobacteria. Microbiology, 2000, 146: 199-208

27 Hunter R L, Olsen M, Jagannath C, et al. Trehalose 6,6'-dimycolate and lipid in the pathogenesis of caseating granulomas of tuberculosis in mice. Am J Pathol, 2006, 168: 1249-1261

28 Ryll R, Kumazawa Y, Yano I. Immunological properties of trehalose dimycolate (cord factor) and other mycolic acid-containing glycolipids - a review. Microbiol Immunol, 2001, 45: 801-811

29 Hunter R L, Venkataprasad N, Olsen M R. The role of trehalose dimycolate (cord factor) on morphology of virulent M. tuberculosis in vitro. Tuberculosis (Edinb), 2006, 86: 349-356

30 Kan-Sutton C, Jagannath C, Hunter R L Jr. Trehalose 6,6'-dimycolate on the surface of Mycobacterium tuberculosis modulates surface marker expression for antigen presentation and costimulation in murine macrophages. Microbes Infect, 2009, 11: 40-48

$31 \mathrm{He} \mathrm{H}$, Hovey $\mathrm{R}$, Kane $\mathrm{J}$, et al. MprAB is a stress-responsive two-component system that directly regulates expression of sigma factors SigB and SigE in Mycobacterium tuberculosis. J Bacteriol, 2006, 188: 2134-2143

32 Pang X, Vu P, Byrd T F, et al. Evidence for complex interactions of stress-associated regulons in an mprAB deletion mutant of Mycobacterium tuberculosis. Microbiology, 2007, 153: 1229-1242

33 Manganelli R, Voskuil M I, Schoolnik G K, et al. The Mycobacterium tuberculosis ECF sigma factor sigmaE: Role in global gene expression and survival in macrophages. Mol Microbiol, 2001, 41: 423437

34 Pang X, Howard S T. Regulation of the alpha-crystallin gene acr 2 by the MprAB two-component system of Mycobacterium tuberculosis. J Bacteriol, 2007, 189: 6213-6221

$35 \mathrm{He} \mathrm{H}$, Zahrt T C. Identification and characterization of a regulatory sequence recognized by Mycobacterium tuberculosis persistence regulator MprA. J Bacteriol, 2005, 187: 202-212

36 Kumar A, Toledo J C, Patel R P, et al. Mycobacterium tuberculosis DosS is a redox sensor and DosT is a hypoxia sensor. Proc Natl Acad Sci USA, 2007, 104: 11568-11573

37 Kendall S L, Movahedzadeh F, Rison S C, et al. The Mycobacterium tuberculosis dosRS two-component system is induced by multiple stresses. Tuberculosis, 2004, 84: 247-255

38 Malhotra V, Sharma D, Ramanathan V D, et al. Disruption of response regulator gene, $\operatorname{dev} R$, leads to attenuation in virulence of $M y$ cobacterium tuberculosis. FEMS Microbiol Lett, 2004, 231: 237-245

39 Converse P J, Karakousis P C, Klinkenberg L G, et al. Role of the dosR-dosS two-component regulatory system in Mycobacterium tuberculosis virulence in three animal models. Infect Immun, 2009, 77: 1230-1237

40 Park H D, Guinn K M, Harrell M I, et al. Rv3133c/dosR is a transcription factor that mediates the hypoxic response of Mycobacterium tuberculosis. Mol Microbiol, 2003, 48: 833-843

41 Walderhaug M O, Polarek J W, Voelkner P, et al. KdpD and KdpE, proteins that control expression of the $k d p A B C$ operon, are members of the two-component sensor-effector class of regulators. J Bacteriol, 1992, 174: 2152-2159

42 Steyn A J, Joseph J, Bloom B R. Interaction of the sensor module of Mycobacterium tuberculosis H37Rv KdpD with members of the $\mathrm{Lpr}$ family. Mol Microbiol, 2003, 47: 1075-1089

43 Haydel S E, Clark-Curtiss J E. Global expression analysis of two-component system regulator genes during Mycobacterium tuberculosis growth in human macrophages. FEMS Microbiol Lett, 2004, 236: 341-347

44 Bacon J, Dover L G, Hatch K A, et al. Lipid composition and transcriptional response of Mycobacterium tuberculosis grown under iron-limitation in continuous culture: Identification of a novel wax ester. Microbiology, 2007, 153: 1435-1444

45 Betts J C, Lukey P T, Robb L C, et al. Evaluation of a nutrient starvation model of Mycobacterium tuberculosis persistence by gene and protein expression profiling. Mol Microbiol, 2002, 43: 717-731

46 DeMaio J, Zhang Y, Ko C, et al. A stationary-phase stress-response sigma factor from Mycobacterium tuberculosis. Proc Natl Acad Sci USA, 1996, 93: 2790-2794

47 Geiman D E, Kaushal D, Ko C, et al. Attenuation of late-stage disease in mice infected by the Mycobacterium tuberculosis mutant lacking the $\mathrm{SigF}$ alternate sigma factor and identification of SigF-dependent genes by microarray analysis. Infect Immun, 2004, 72: 1733-1745

48 Dainese E, Rodrigue S, Delogu G, et al. Posttranslational regulation of Mycobacterium tuberculosis extracytoplasmic-function sigma factor sigma $\mathrm{L}$ and roles in virulence and in global regulation of gene expression. Infect Immun, 2006, 74: 2457-2461

49 Williams E P, Lee J H, Bishai W R, et al. Mycobacterium tuberculosis $\mathrm{SigF}$ regulates genes encoding cell wall-associated proteins and directly regulates the transcriptional regulatory gene phoY1. J Bacteriol, 2007, 189: 4234-4242

50 Raman S, Song T, Puyang X, et al. The alternative sigma factor SigH regulates major components of oxidative and heat stress responses in Mycobacterium tuberculosis. J Bacteriol, 2001, 183: 6119-6125

51 Manganelli R, Voskuil M I, Schoolnik G K, et al. Role of the extracytoplasmic-function sigma factor $\operatorname{sigma}(\mathrm{H})$ in Mycobacterium tuberculosis global gene expression. Mol Microbiol, 2002, 45: 365-374

52 Mulder N J, Zappe H, Steyn L M. Characterization of a Mycobacterium tuberculosis homologue of the Streptomyces coelicolor whiB gene. Tuber Lung Dis, 1999, 79: 299-308

53 Singh A, Guidry L, Narasimhulu K V, et al. Mycobacterium tuberculosis WhiB3 responds to $\mathrm{O} 2$ and nitric oxide via its [4Fe-4S] cluster and is essential for nutrient starvation survival. Proc Natl Acad Sci USA, 2007, 104: 11562-11567

54 Singh A, Crossman D K, Mai D, et al. Mycobacterium tuberculosis WhiB3 maintains redox homeostasis by regulating virulence lipid anabolism to modulate macrophage response. PLoS Pathog, 2009, 5: e1000545

55 Banaiee N, Jacobs W R Jr, Ernst J D. Regulation of Mycobacterium tuberculosis whiB3 in the mouse lung and macrophages. Infect Immun, 2006, 74: 6449-6457

56 Avarbock D, Salem J, Li L S, et al. Cloning and characterization of a bifunctional RelA/SpoT homologue from Mycobacterium tuberculosis. Gene, 1999, 233: 261-269

57 Dahl J L, Kraus C N, Boshoff H I, et al. The role of RelMtb-mediated adaptation to stationary phase in long-term persistence of Mycobacterium tuberculosis in mice. Proc Natl Acad Sci USA, 2003, 100: 10026-10031

58 Cunningham A F, Spreadbury C L. Mycobacterial stationary phase induced by low oxygen tension: Cell wall thickening and localization of the 16-kilodalton alpha-crystallin homolog. J Bacteriol, 1998, 180: 801-808

59 Yuan Y, Crane D D, Barry C E. Stationary phase-associated protein expression in Mycobacterium tuberculosis: Function of the mycobacterial alpha-crystallin homolog. J Bacteriol, 1996, 178: 44844492

60 Sureka K, Dey S, Datta P, et al. Polyphosphate kinase is involved in stress-induced mprAB-sigE-rel signalling in mycobacteria. Mol Microbiol, 2007, 65: 261-276

61 Domenech P, Reed M B, Barry C E. Contribution of the Mycobacterium tuberculosis $\mathrm{MmpL}$ protein family to virulence and drug resistance. Infect Immun, 2005, 73: 3492-3501

62 Romero I C, Mehaffy C, Burchmore R J, et al. Identification of promoter-binding proteins of the $f b p A$ and $C$ genes in Mycobacterium tuberculosis. Tuberculosis (Edinb), 2010, 90: 25-30

63 Lee J H, Karakousis P C, Bishai W R. Roles of SigB and SigF in the Mycobacterium tuberculosis sigma factor network. J Bacteriol, 2008, 190: 699-707

64 Fontan P A, Voskuil M I, Gomez M, et al. The Mycobacterium tuberculosis sigma factor sigmaB is required for full response to cell envelope stress and hypoxia in vitro, but it is dispensable for in vivo growth. J Bacteriol, 2009, 191: 5628-5633

65 Lin M Y, Ottenhoff T H. Not to wake a sleeping giant: New insights into host-pathogen interactions identify new targets for vaccination 
against latent Mycobacterium tuberculosis infection. Biol Chem, 2008, 389: 497-511

66 Geluk A, Lin M Y, van Meijgaarden K E, et al. T-cell recognition of the HspX protein of Mycobacterium tuberculosis correlates with latent $M$. tuberculosis infection but not with $M$. bovis BCG vaccination. Infect Immun, 2007, 75: 2914-2921

67 Lowrie D B, Tascon R E, Bonato V L, et al. Therapy of tuberculosis in mice by DNA vaccination. Nature, 1999, 400: 269-271

68 Flores Valdez M A, Schoolnik G K. DosR-regulon genes induction in
Mycobacterium bovis BCG under aerobic conditions. Tuberculosis, 2010, 90: 197-200

69 Murphy D J, Brown J R. Novel drug target strategies against Mycobacterium tuberculosis. Curr Opin Microbiol, 2008, 11: 422-427

70 Gomez J E, McKinney J D. M. tuberculosis persistence, latency, and drug tolerance. Tuberculosis, 2004, 84: 29-44

71 Kong Y, Yao H, Ren H, et al. Imaging tuberculosis with endogenous $\beta$-lactamase reporter enzyme fluorescence in live mice. Proc Natl Acad Sci USA, 2010, 107: 12239-12244

Open Access This article is distributed under the terms of the Creative Commons Attribution License which permits any use, distribution, and reproduction in any medium, provided the original author(s) and source are credited. 